\title{
Molecular Cloning, Characterization and Expression of SUPPRESSOR of OVEREXPRESSION of CONSTANS 1 (NnSOC1) and NnSOC1-like in Nelumbo nucifera
}

\author{
Chen Dong ${ }^{1 *}$, Fei $\mathrm{Du}^{1}$, Ye $\mathrm{Li}^{2}$, Ningning Yang ${ }^{1}$, Jiaqi $\mathrm{Mao}^{1}$ and Zhongli $\mathrm{Hu}^{2}$ \\ ${ }^{1}$ College of Biological Engineering, Henan University of Technology, Zhengzhou, Henan 450001, China \\ ${ }^{2}$ State Key Laboratory of Hybrid Rice, Lotus Engineering Research Center of Hubei Province, College of Life Science, Wuhan \\ University, Wuhan 430072, China \\ *For correspondence: chen.dong@haut.edu.cn \\ Received 09 September 2020; Accepted 24 December 2020; Published 25 March 2021
}

\begin{abstract}
For the aim of unveiling the molecular mechanism of flowering, the MADS-box genes of SUPPRESSOR of OVEREXPRESSION of CONSTANS 1 (NnSOC1) and NnSOC1-like were isolated in Nelumbo nucifera. Seven introns splicing of $\mathrm{NnSOCl}$ and $\mathrm{NnSOCl}$-like strictly followed the GT-AG rule, consisting of all the characteristic motifs of SOC1 family. $\mathrm{NnSOCl}$ and $\mathrm{NnSOCl-like}$ were widely distributed in reproductive and vegetative tissues of $\mathrm{N}$. nucifera, exhibiting the highest expression in leaves and the lowest level in embryo. Additionally, both genes were expressed in the whole flowering stage, with the highest mRNA level observed in the initiation stage of flowering and the lowest expression in fruit set. Ectopic expression of $\mathrm{NnSOCl}$ and $\mathrm{NnSOCl}$-like advanced the flowering time of transgenic Arabidopsis, and decreased the rosetta leaves production. These results suggested that NnSOCl and NnSOC1-like were involved in initiation of flowering, which are likely to serve as fundamental research for studying molecular mechanism of flowering in aquatic plants. (C) 2021 Friends Science Publishers
\end{abstract}

Key words: Flower opening; Gene expression; MADS-box; Nelumbo nucifera; SOC1

\section{Introduction}

Flowering is the floral transformation from vegetative phase to reproductive stage, which is considered as an imperative agronomic trait of crops. The floral initiation is critical for harvesting more products, controlling by combined function of endogenous gene network as well as environmental factors. Unveiling the molecular mechanism of flowering would be benefit for breeding in various surroundings. Various signals were integrated by networks to determine flower transition. Up to date, at least four flowering pathways including long day, autonomous, vernalization and gibberellin pathways were reported to regulate floral induction of high plants (Liu et al. 2012). MADS-box genes, encoding a highly conserved domain known as MADS-box, were considered as the key members of gene networks controlling the flowering transition and development.

The SUPPRESSOR of OVEREXPRESSION of CONSTANS 1 (SOC1) was classified as MADS-box type I $\left(\mathrm{MIKC}^{\mathrm{C}}\right.$ ) in high plants (Lee and Lee 2010), and is considered as a critical integrator for flowering activation in Arabidopsis. SOC1 integrated various flowering signals from temperature, photoperiod and hormones. As a key composition of transcription factors, SOC1 was characterized by the domains of MADS-box (M), an intervening region (I), a keratin box $(\mathrm{K})$, as well as a $\mathrm{C}$ terminal domain (C) (Zhong et al. 2012; Li et al. 2020). SOC1 genes from higher plants including peony, barley, apricot, soybean and Mango have been studied (Papaefthimiou et al. 2012; Zhong et al. 2012; Wang et al. 2015; Wei et al. 2016), which were highly conserved among angiosperms. SOCl genes were widely distributed in tissues of leaves, flower and root. Ectopic expression of SOCI stimulated the early flowering of tobacco, tree peony and Pyrus bretschneideri (Wang et al. 2015; Yu et al. 2020; Liu et al. 2020). Beside flowering time, SOC1 also had other biological roles such as regulation of petal development in Gerbera hybrida (Ruokolainen et al. 2011), and floral organ senescence in Camellia sinensis (Tan and Swain 2007).

Lotus (Nelumbo nucifera L.) belongs to Nelumbonaceae family, known as a perennial aquatic plant. $N$. nucifera was famous as imperative ornamental plant as well as economic crop, having colorful flowers and numbers of petals. N.nucifera is considered as the species between dicots and monocots (Yang et al. 2014). The flowers, leaves, seeds and buds appear at the reproductive stage

To cite this paper: Dong C, F Du, Y Li, N Yang, J Mao, Z Hu (2021). Molecular cloning, characterization and expression of suppressor of OVEREXPRESSION of CONSTANS 1 (NnSOC1) and NnSOC1-like in Nelumbo nucifera. Intl J Agric Biol 25:851-858 
of $N$. nucifera. Although SOC1 family was relatively conserved, similar expression pattern was detected in dicots and monocots, their function might be divergent in $N$. nucifera. Therefore, further studies are necessary to unveil the functional divergence of SOCl for the aim of understanding the special mechanism controlling initiation of flowering in $N$. nucifera.

In this study, $\mathrm{NnSOCl}$ and $\mathrm{NnSOCl}$-like cDNAs in $\mathrm{N}$. nucifera were isolated. Their exon-intron structures, conserved motifs and phylogenetic analysis were performed. The three-dimensional structures were generated by homology modeling. Additionally, the expression pattern of both genes was examined during flower opening. Finally, the function of $\mathrm{NnSOCl}$ and NnSOCl-like were illustrated by transferring into the wild type Arabidopsis.

\section{Materials and Methods}

\section{Plant material}

Nelumbo nucifera "var. Taikonglian-36" was planted in Henan University of Technology, China. The rhizomes of the same size were taken randomly, and planted in pools with 2 rhizomes in each pool in April, 2018. The size of pools was about $10 \mathrm{~m} \times 5 \mathrm{~m}$. All the samples were harvested at reproductive stage in September 2018, and frozen in liquid nitrogen immediately. Seeds of wild-type and transgenic A. thaliana were germinated and grown in the growth chamber under long-day conditions at $22^{\circ} \mathrm{C}$, with 16 $\mathrm{h}$ light $/ 8 \mathrm{~h}$ darkness cycles and $60 \%$ relative air humidity.

\section{Isolation and characteristic of $\mathrm{NnSOC1}$ and NnSOC1-like}

To scan SOCl in the genome of $N$. nucifera, the key word "SOCl" was used to search the Sacred lotus (N. nucifera) genome database (Ming et al. 2013). The putative proteins were further searched in the NCBI conserved domain to confirm the presence of conserved domains of SOC1. The primers were used to isolate the complete open reading frames (ORF) of $\mathrm{NnSOCl}$ and NnSOCl-like respectively (Table 1), based on their nucleotide sequences (XM_010257287; XM_010274299). The first leaf was collected in vegetative stage and frozen in liquid nitrogen immediately. Total RNAs from the first leaf were isolated by RNAprep pure Plant kit (TIANGEN, China). The first strand cDNA was generated by M-MLV transcriptase (Promega, USA) by reverse transcription. The reaction mixture was as follow: $3 \mu \mathrm{L}$ RNA, $2 \mu \mathrm{L}$ olig(dT) $)_{17}, 5 \mu \mathrm{L}$ M-MLV reaction buffer, $3 \mu \mathrm{L}$ dNTP, $10 \mu \mathrm{L}$ nuclease-free water and $2 \mu \mathrm{L}$ MMLV transcriptase. The target products were harvested and sequenced as described previously (Dong et al. 2015).

\section{Characterization and exon-intron structures}

The Prot Param program was performed to evaluate the putative molecular weight (MW) and isoelectric point (pI), based on amino acid compositions of $\mathrm{NnSOC1}$ and
NnSOC1-like. Protcomp Version 9.0 software was used to predict the Sub-cellular location. Protein structures of NnSOC1 and NnSOC1-like were predicted by SMART online tools. Conserved motifs were indicated using online MEME program. The exon-intron structures of both genes were analyzed by Gene Structure Display Server (GSDS).

\section{Similarity and the homology model of NnSOC1 and NnSOC1-like}

Multiple alignment of the putative amino acid sequences of NnSOC1 and NnSOC1-like with other SOC1 from higher plants was performed by CLUSTAL W. Phylogenetic analysis was carried out by the Neighor-Joining method using the software of MEGA version 4 (Tamura et al. 2007). In order to further characterize their structures, Myocytespecific enhancer factor 2B (MEF2B) from Homo sapiens (PDB No. 1n6j) was selected as the highest scoring template for homology model (Han et al. 2003). The homology models of $\mathrm{NnSOC1}$ and $\mathrm{NnSOC1-like} \mathrm{were} \mathrm{constructed,}$ using phyre2 bath processing (Kelley et al. 2015).

\section{The expression profile of $\mathrm{NnSOC1}$ and NnSOC1-like}

Eight-weeks old lotus was planted in pool, and the total RNA was isolated from embryo, flower, root, stem and leaves in vegetative stage for investigating their expression in various tissues. Moreover, the leaves were collected during the four stages of flowering with the reported method (Yang et al. 2014). Four stages were: Stage 1, floral buds were generated underwater; Stage 2, floral buds emerged from water; Stage 3, floral buds developed into bloom; Stage 4 , the flowers were pollinated and seeds were produced. Real-time PCR was performed for detection of $\mathrm{NnSOCl}$ and $\mathrm{NnSOCl}$-like. Relative expression of $\mathrm{NnSOCl}$ and NnSOCl-like was calculated using $\beta$-actin as the reference gene (Livak and Schmittgen 2001).

\section{Ectopic expression of $\mathrm{NnSOC1}$ and NnSOC1-like in Arabidopsis}

For further exploring the roles of $\mathrm{NnSOCl}$ and $\mathrm{NnSOCl}$ like in flower opening, both ORF amplified by PCR using gene-specific primers including attB-sites (Table 1), were inserted into pEarleyGate 101 (Earley et al. 2006) using the Gateway LR reaction (Invitrogen). The Agrobacterium tumefaciens GV3101 strain electroporated by recombinant plasmids and was transformed into wild type Arabidopsis. Transformed Arabidopsis seeds were selected by spraying a $0.002 \%(\mathrm{~V} / \mathrm{V})$ Basta solution, and T3 homozygote plants were used for further experiments. The flowering phenotype of transgenic Arabidopsis was examined.

\section{Statistical analysis}

Three independent experiments were performed to ensure 
Table 1: Primers used in the present study

\begin{tabular}{|c|c|c|}
\hline Primer name & Sequence (5'-3') & Experiments \\
\hline $\mathrm{NnSOC1} \mathrm{CF}$ & ATGGTGAGGGGGAAGACCCAGATGA & Isolation $\mathrm{NnSOCl}$ \\
\hline NnSOC1 CR & TCATACTGAGCCATCTCCAACCAAT & Isolation $\mathrm{NnSOCl}$ \\
\hline NnSOC1-like CF & ATGGTGAGGGGGAAGACGCAGATGA & Isolation $\mathrm{NnSOC1-like}$ \\
\hline NnSOC1-like CR & CTAATAGTCCTGTAATGGGTAGCGT & Isolation $\mathrm{NnSOC1-like}$ \\
\hline NnSOC1 F & TTATTTAGGGAGCAGATTGCAA & Real-time PCR \\
\hline NnSOC1 R & TTATTCAGGGAGCAGATTGAGG & Real-time PCR \\
\hline NnSOC1-like F & GCTCTTTCAGGCCTCCCA & Real-time PCR \\
\hline NnSOC1-like R & GCTCTTTCAGGCCTCCCT & Real-time PCR \\
\hline$\beta$-actin F & TGATCGGAATGGAAGC & Real-time PCR \\
\hline$\beta$-actin R & CAGCAATACCAGGGAAC & Real-time PCR \\
\hline $\mathrm{NnSOC} 1 \mathrm{EF}$ & ggggacaagtttgtacaaaaaagcaggctATGGTGAGGGGGAAGACCCA & Ectopic expression of $\mathrm{NnSOCl}$ \\
\hline NnSOC1 ER & ggggaccactttgtacaagaaagctgggtaTACTGAGCCATCTCCAACCAAT & Ectopic expression of $\mathrm{NnSOCl}$ \\
\hline NnSOC1-like EF & ggggacaagtttgtacaaaaaagcaggctATGGTGAGGGGGAAGACGCA & Ectopic expression of $\mathrm{NnSOCl}$-like \\
\hline NnSOC1-like ER & ggggaccactttgtacaagaaagctgggtaATAGTCCTGTAATGGGTAGC & Ectopic expression of $\mathrm{NnSOCl}$-like \\
\hline
\end{tabular}

reproducibility. Data were expressed as the mean \pm SD from three independent biological replicates. Significance was calculated based on one-way analysis of variance (ANOVA) by SPSS 22.0 software. Different letters represent significant differences at $\mathrm{p}<0.05$.

\section{Results}

\section{Identification and conserved domains of $\mathrm{NnSOC1}$ and NnSOC1-like}

The ORF of NnSOC1 and NnSOCl-like was 675 and $654 \mathrm{bp}$ respectively, consisting of the start codon ATG and stop codon TAG. NnSOC1 mRNA encoded a putative protein of 224 amino acids, with predicted MW of $25.68 \mathrm{kD}$ and $\mathrm{pI}$ of 9.37. The putative protein of NnSOC1-like was composed of 217 amino acids with MW of $25.41 \mathrm{kD}$ and $\mathrm{pI}$ of 9.28. Both had the similar structure of the MADS-box family, consisting of highly conserved MADS-box, variable I-box, relative conserved K-box with the size of 93 amino acid residues and variable $\mathrm{C}$-terminal domain (Fig. 1). The SOC1 motif (DVETELFIGRP) was highly conserved in the C-terminal of NnSOC1 and NnSOC1-like.

\section{Exon-intron architectures of $\mathrm{NnSOC1}$ and $\mathrm{NnSOC1-}$ like genes}

Exon-intron architectures of NnSOCl and NnSOCl-like genes were almost the same, consisting of 8 exons and 7 introns. Their ORF contained nucleotide sequences of partial exon 2 , exon 3 , exon 4 , exon 5 , exon 6 , exon 7 and partial exon 8 . The sizes of exon 3 (79 bp), exon 4 (62 bp), exon 5 (100 bp), exon 6 (42 bp) and exon 7 (42 bp) were the same in NnSOCl and NnSOCl-like, with little difference in exon 1 (382 bp for $\mathrm{NnSOCl;} 329 \mathrm{bp}$ for NnSOC1-like), exon 2 (190 bp for NnSOC1; $252 \mathrm{bp}$ for NnSOCl-like) and exon 8 (423 bp for NnSOCl; 402 bp for NnSOCl-like). The introns splicing of NnSOCl and NnSOCl-like genes strictly followed the GT-AG rule (Fig. 2).
The phylogenetic analysis of NnSOC1 and NnSOC1-like

A phylogenetic tree was constructed based on the amino acid sequences of high plants, indicating all the SOC 1 could be grouped into dicot and monocot clades (Fig. 3). The SOC1 of Triticum aestivum (TaSOC1) and Hordeum vulgare (HvSOC1) from monocot was grouped into one branch, whereas SOC1 and SOC1-like from higher plants were clustered together. Although NnSOC1 and NnSOC1like were grouped into dicot (Fig. 3), they had relatively farther relationship with other members in dicot. The special motif (motif 10) known as MEHPNQN was detected in both NnSOC1 and NnSOC1-like (Fig. 3).

\section{The homology model of NnSOC1 and NnSOC1-like}

About $41 \%$ of residues in NnSOC1 and $42 \%$ of residues in NnSOC1-like were modeled with $100 \%$ confidence. Both NnSOC1 and NnSOC1-like were consisted of two spatially distinct domains, the $\alpha \beta \beta \alpha$ structure of the MADS-box (AA 13-71), and the large helical K-box (Fig. 4). About 59\% $\alpha$ helix was widely detected in the amino acid sequence of NnSOC1, which were inlaid by $5 \% \beta$-strand. Moreover, $\alpha$ helix in NnSOC1-like was about $57 \%$ with $5 \% \beta$-strand.

\section{Expression profile of NnSOC1 and NnSOC1-like in various tissues}

The expression pattern of two transcripts was studied in reproductive and vegetative tissues by Real-time PCR. NnSOCl and NnSOCl-like mRNAs were widely distributed in root, leave, stem, flower and embryo, exhibiting the similar expression pattern (Fig. 5). The expression of NnSOC1 mRNA in leave (8.46 fold), stem (7.41 fold), root (5.29 fold) and flower (4.11 fold) was significantly more than embryo. Additionally, the mRNA level of $\mathrm{NnSOC1}$ like in leave (3.23 fold), stem (2.91 fold) and root (1.94 fold) was relatively higher than flower and embryo (Fig. 5). The mRNA level of $\mathrm{NnSOCl}$ was relatively higher than NnSOCl-like, suggesting NnSOCl was the major transcript in the tissues examined. 


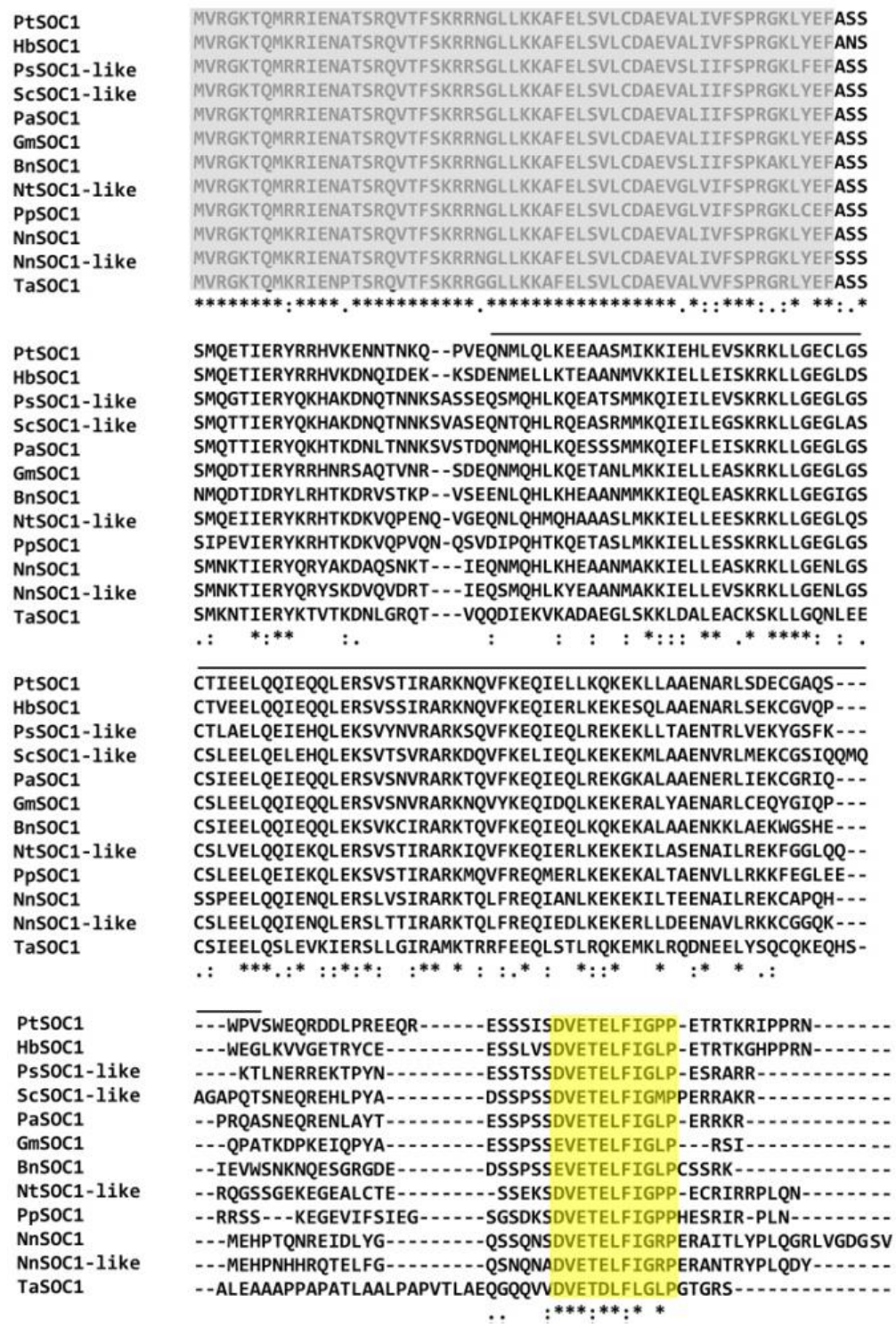

Fig. 1: Amino acid sequence alignment and characteristics of NnSOC1 and NnSOC1-like. The highly conserved MADS-box was shaded in grey, and relative conserved K-box was lined. The highly conserved SOC1 motif (DVETELFIGRP) was represented by yellow in the C-terminal of proteins

\section{The expression pattern of $\mathrm{NnSOC1}$ and $\mathrm{NnSOC1-like}$ in} flower opening

Real-time PCR indicated that NnSOCl and NnSOCl-like mRNAs were decreased in the process of flower opening (Fig. 6). Both transcripts showed the highest mRNA level in leaves when floral buds were generated underwater (stage 1). NnSOCl started to decrease during the stage of floral buds appearing from water, and it was relatively stable at the stage of developing into bloom (stage 3). Then NnSOC1 mRNA was further decreased into the lowest mRNA level when the flowers were pollinated and plant yielded fruit (stage 4). Interestingly, NnSOCl-like exhibited the similar expression pattern with $\mathrm{NnSOC1}$. NnSOCl-like mRNA at stage 1was almost the same to stage 2. Then NnSOC1-like was significantly down-regulated at stage 3 , which was further declined to the lowest level at stage 4. Moreover, NnSOC1 mRNA exhibited higher expression level than NnSOC1-like.

Functional analysis of $\mathrm{NnSOC1}$ and NnSOC1-like in transgenic arabidopsis

To examine the role of NnSOCl and NnSOCl-like in regulation of flowering, they were overexpressed in Arabidopsis by the CaMV $35 \mathrm{~S}$ promoter. The early flowering phenotype was detected in the transgenic lines of 35 S::NnSOC1 and 35 S::NnSOCl-like (Fig. 7a). NnSOC1 

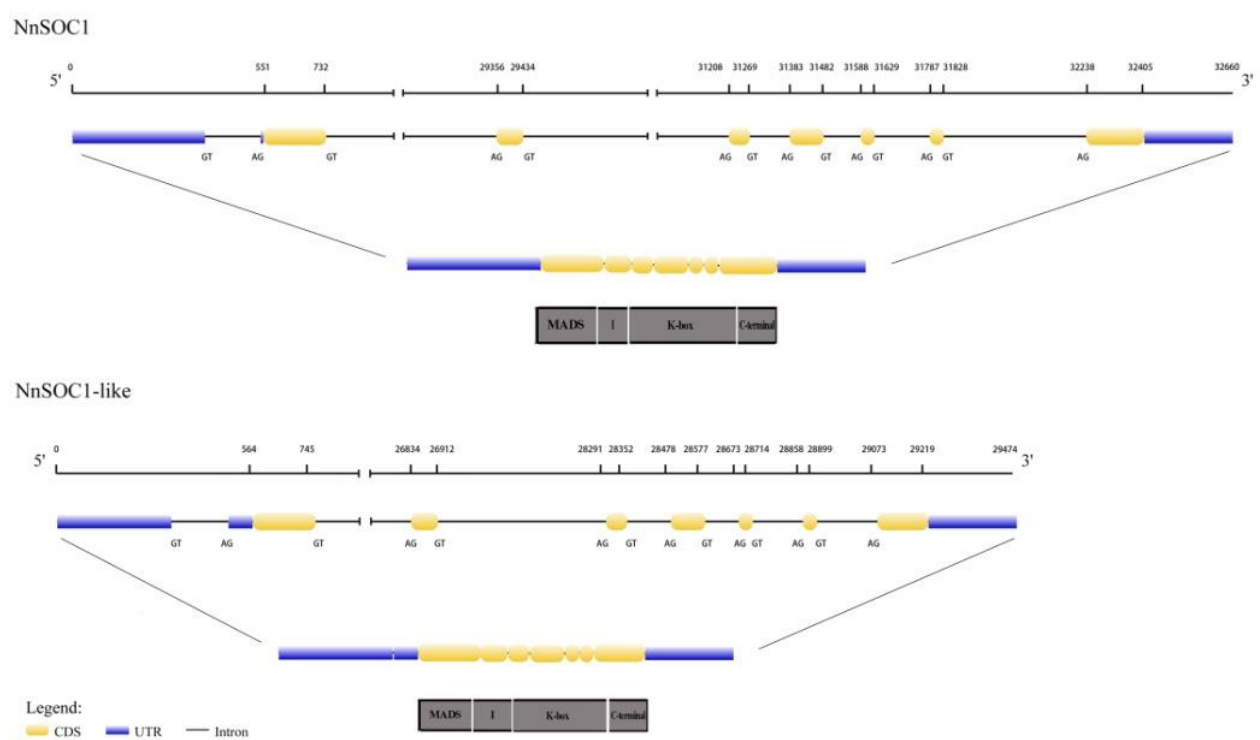

Fig. 2: Exons-intron architecture of NnSOCl and NnSOCl-like genes. The UTR, CDS and introns were labeled. The highly conserved MADS-box, variable I-box, relative conserved K-box and variable C-terminal domains were represented
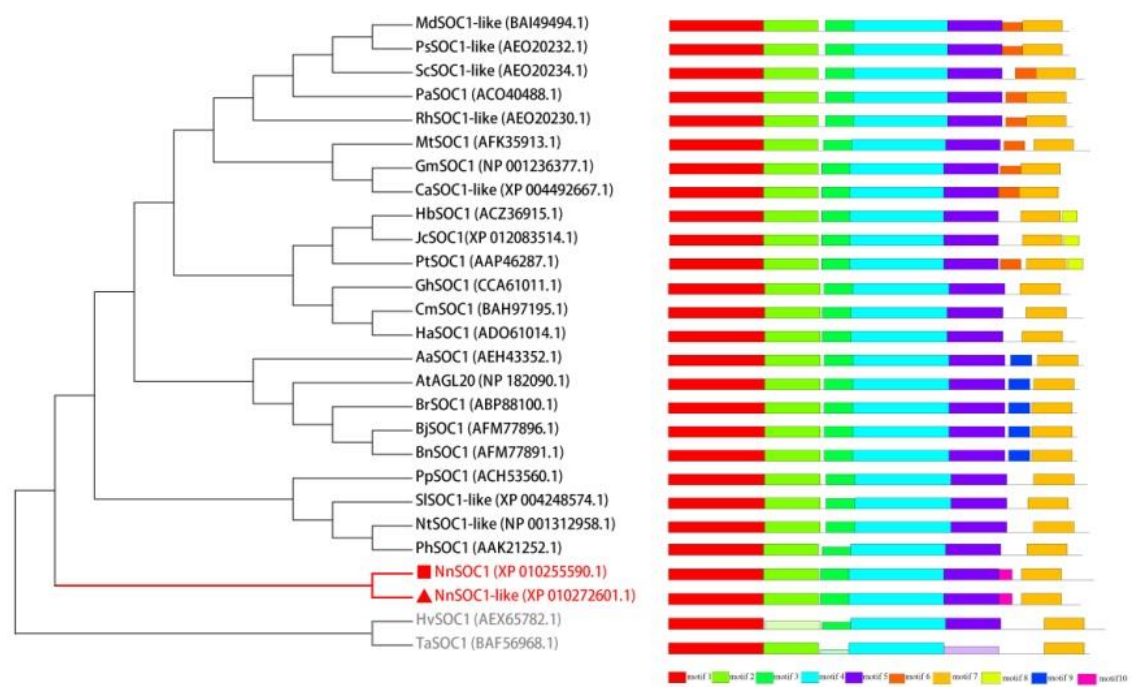

Fig. 3: The conserved motifs and phylogenetic relationship of SOC1 family. Phylogenetic analysis of SOC1 family was performed by MEGA4 software. The special motif (motif 10) known as MEHPNQN was detected in both NnSOC1 and NnSOC1-like

and NnSOC1-like advanced the flowering time of transgenic lines, with 21 days earlier than wild type (Fig. 7b). However, ectopic expression of NnSOCl and NnSOCl-like decreased the number of rosette leaves, and more fewer rosette leaves were detected in $35 \mathrm{~S}:: \mathrm{NnSOCl-like}$ than 35 S::NnSOCl (Fig. 7b).

\section{Discussion}

Considering as one of the most important aquatic crops widely planted in tropic and subtropic regions, the molecular mechanism of the floral transition in $N$. nucifera are still unveiled. As one of the most important members of
MADS-box genes, NnSOCl and NnSOCl-like were identified and their amino acid sequences were characterized in this study. MADS-box domain and K-box domain were identified in NnSOC1 and NnSOC1-like (Fig. 1), which were considered as two characteristic domains of MADS-box family (Ruokolainen et al. 2011; Ding et al. 2013; Zheng et al. 2020). Therefore, NnSOC1 and NnSOC1-like were classified as the members of MADSbox family (Ding et al. 2013). Three amino acid residues $\left(\mathrm{Arg}^{24}, \mathrm{Glu}^{34}\right.$ and $\mathrm{Gly}^{112}$ ) in NnSOC1 and NnSOC1-like were the same with SOC1 from Arabidopsis, and substitution of these residues in Arabidopsis could affect early flowering time (Lee et al. 2008). 

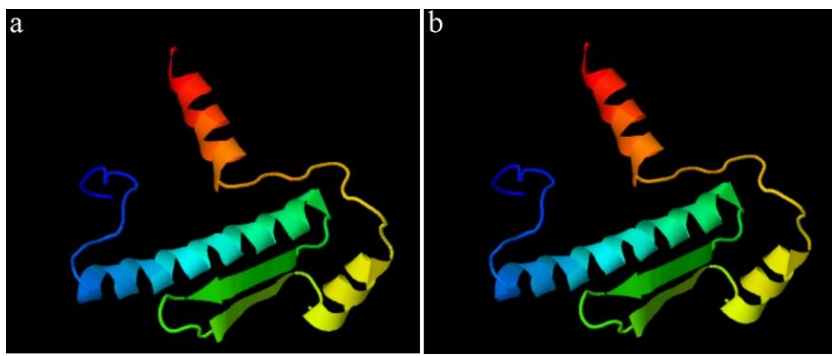

Fig. 4: The three-dimensional structures of MADS-box domains of the NnSOC1 (a) and NnSOC1-like (b)

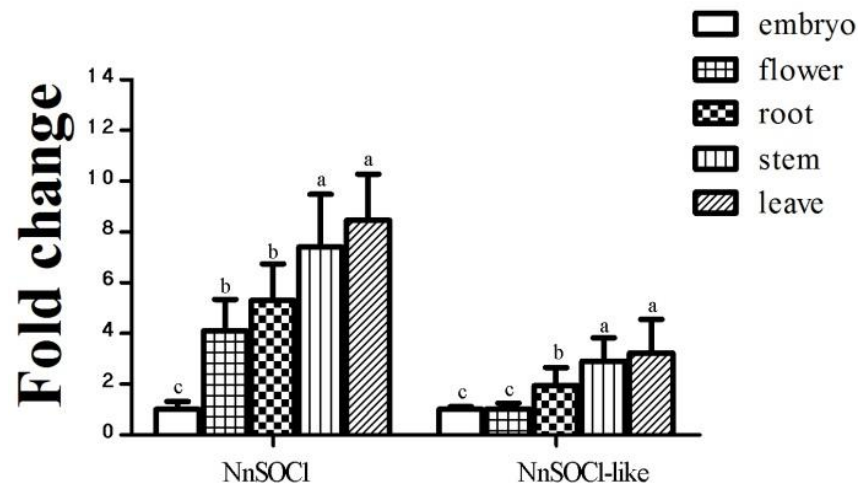

Fig. 5: The expression of NnSOCl and NnSOCl-like in various tissues. Real-time PCR was performed to test the mRNA level of $\mathrm{NnSOCl}$ and NnSOCl-like in embryo, leaves, flower, stem and root

Data were expressed as the mean \pm SD from three independent biological replicates. Significance was calculated based on one-way ANOVA analysis by SPSS 22.0 software. Different letters represent significant differences at $\mathrm{p}<0.05$

a
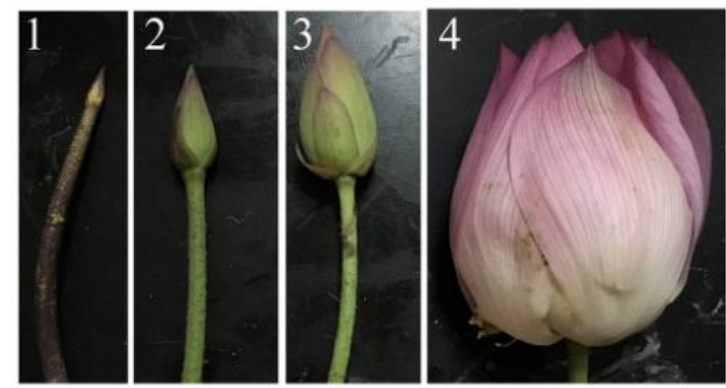

$\mathrm{b}$

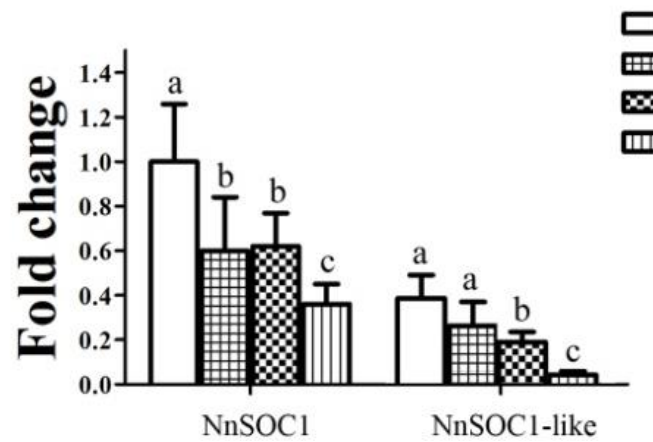

Fig. 6: Expression pattern of $\mathrm{NnSOCl}$ and $\mathrm{NnSOCl-like} \mathrm{during} \mathrm{the} \mathrm{four} \mathrm{stages} \mathrm{of} \mathrm{flowering.} \mathrm{The} \mathrm{total} \mathrm{RNAs} \mathrm{were} \mathrm{extracted} \mathrm{from} \mathrm{leave}$ during the life cycle of flower (a). And NnSOCl and NnSOCl-like mRNAs were examined by Real-time PCR (b)

The exons-intron architecture of NnSOCl and NnSOCl-like genes was almost the same, following the GT-
AG rule (Fig. 2). Moreover, SOC1 and SOC1-like in high plants were analyzed by Neighbor-Joining method, which 

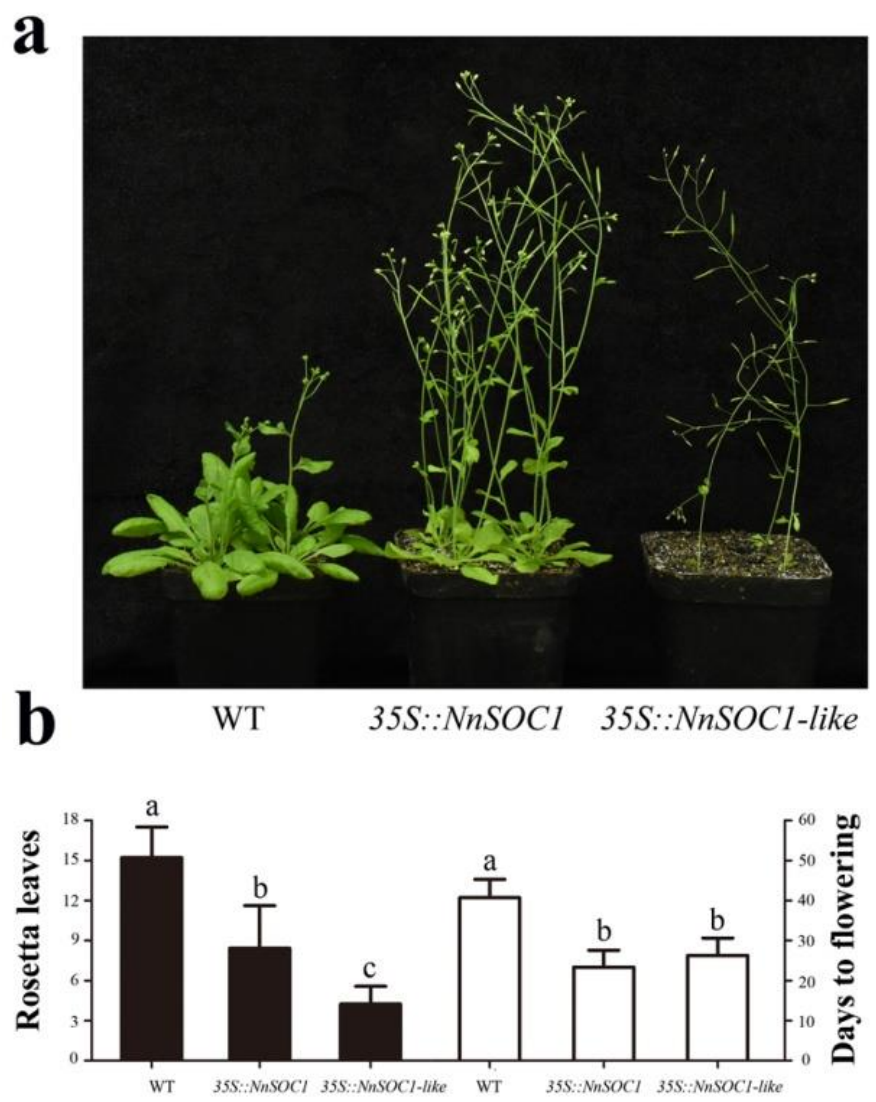

Fig. 7: Ectopic expression of NnSOCl and NnSOCl-like. The phenotype of NnSOCl and NnSOCl-like transgenic lines (a). Days to flowering and the counts of rosette leaves for 35S::NnSOC1 and 35S::NnSOC1-like transgenic lines (b) Data were calculated from three independent experiments, using different letters represented significant difference $(\mathrm{p}<0.05)$

indicated that NnSOC1 and NnSOC1-like had more homology with SOC1 and SOC1-like of dicot (Fig. 3). It suggested that SOC1 of higher plants might have come from the same ancestor before evolving independently in dicots and monocots (Wei et al. 2016).

The SOCl genes were expressed in distinct tissues, showing various roles in dicot plants (Borner et al. 2000). Our results indicated both $\mathrm{NnSOCl}$ and $\mathrm{NnSOCl}$-like were widely expressed in reproductive and vegetative tissues (Fig. 5), while more expression of both genes was found in leave than flower. This was consistent with the study in Arabidopsis, $O$. sativa, $H$. vulgare and T. aestivum (Komiya et al. 2009; Papaefthimiou et al. 2012). The SOC1 was mainly considered as the integrator of multiple flower signals. $\mathrm{NnSOCl}$ and $\mathrm{NnSOCl}$-like mRNAs were decreased in the process of flower opening. The highest expression was detected in the leaves of floral initiation, and the least mRNA level was found at fruit set (Fig. 6). The downregulation of $\mathrm{SOCl}$ during flower opening was also reported in Mango (Wei et al. 2016).

Overexpression of $\mathrm{NnSOCl}$ and NnSOCl-like significantly promoted early flowering (Fig. 7). This suggested that the role of flowering activator for SOC1 was almost conserved in the high plants (Ding et al. 2013; Wang et al. 2015). Moreover, the abnormal leaves were observed in 35S::NnSOCl and 35S::NnSOCl-like transgenic Arabidopsis, which were also detected in transgenic Arabidopsis by overexpression of SOCl genes in mango (Wei et al. 2016), Phyllostachys violascens (Liu et al. 2016) and Davidia involucrate (Li et al. 2020). Although how MADS-box genes regulate floral initiation remains to be elucidated, nonetheless the results provide valuable information for unveil the molecular mechanism of $\mathrm{NnSOCl}$ and $\mathrm{NnSOCl}$-like for regulation the initiation of flower opening.

\section{Conclusion}

Results suggest that $\mathrm{NnSOCl}$ and NnSOCl-like were involved in initiation of flowering in $N$. nucifera. This provides a foundation for breed of this species for horticultural purpose to regulate flowering time in aquatic plants.

\section{Acknowledgments}

This work was supported by National Nature Science Foundation of China (no. 31601366), Science and Technology Planning Project of Henan Province, China (no. 
192102110120), and Natural Science Foundation of Henan Province (202300410114).

\section{Author Contributions}

Chen Dong designed the experiments and wrote the manucript. Fei Du and Ye Li performed Real-time PCR. Ningning Yang isolated SOC1 gene. Jiaqi Mao expressed SOC1 and SOC1-like in Arabidopsis. Zhongli $\mathrm{Hu}$ revised the manuscript. All authors have read and approved the final manuscript.

\section{Conflict of Interest}

There is no conflict of interest among the authors and the institutions where the research has been conducted

\section{Data Availability Declaration}

All data related to this article are in the custody of corresponding author and will be available on request

\section{Ethics Approval}

Not applicable

\section{References}

Borner R, G Kampmann, J Chandler, R Gleissner, E Wisman, K Apel (2000). A MADS domain gene involved in the transition to flowering in Arabidopsis. Plant J 24:591-599

Ding L, Y Wang, H Yu (2013). Overexpression of DOSOC1, an ortholog of ArabidopsisSOC1, promotes flowering in the orchid Dendrobium Chao Parya Smile. Plant Cell Physiol 54:595-608

Dong C, X Zheng, Y Diao, Y Wang, M Zhou, Z Hu (2015). Molecular cloning and expression analysis of a catalase gene (NnCAT) from Nelumbo nucifera. Appl Biochem Biotechnol 177:1216-1228

Earley KW, JR Haag, O Pontes, K Opper, T Juehne, KM Song (2006). Gateway-compatible vectors for plant functional genomics and proteomics. Plant J 45:616-629

Han A, F Pan, JC Stroud, HD Youn, JO Liu, L Chen (2003). Structural basis of Sequence-specific recruitment of transcriptional co-repressor Cabin1 by myocyte enhancer factor-2. Nature 422:730-734

Kelley LA, S Mezulis, CM Yates, MN Wass, MJE Sternberg (2015). The Phyre2 web portal for protein modeling, prediction and analysis. Nat Protoc 10:845-858

Komiya R, S Yokoi, K Shimamoto (2009). A gene network for long-day flowering activates RFT1encoding a mobile flowering signal in rice. Development 136:3443-3450

Lee J, I Lee (2010). Regulation and function of SOC1, a flowering path-way integrator. J Exp Bot 61:2247-2254
Lee J, M Oh, H Park, I Lee (2008). SOC1 translocated to the nucleus by interaction with AGL24 directly regulates leafy. Plant J 55:832-843

Liu S, T Ma, L Ma, X Lin (2016). Ectopic expression of PvSOC1, a homolog of SOC1 from Phyllostachys violascens, promotes flowering in Arabidopsis and rice. Acta Physiol Plantarum 38; Article 166

Li GL, CX Cao, P Gao, Y Zhao (2020). Molecular cloning and potential role of DiSOC1s in flowering regulation in Davidia involucrata Baill. Plant Physiol Biochem 157:453-459

Liu TK, Y Li, CW Zhang, Y Qian, Z Wang, XL Hou (2012). Overexpression of FLOWERING LOCUS C, isolated from NonHeading Chinese cabbage (Brassica campestrisssp. chinensis Makino), influences fertility in Arabidopsis. Plant Mol Biol Rep 30:1444-1449

Liu Z, XP Wu, MY Cheng, ZH Xie, CL Xiong, SL Zhang, JY Wu, P Wang (2020). Identification and functional characterization of SOC1-like genes in Pyrus bretschneideri. Genomics 112:1622-1632

Livak KJ, TD Schmittgen (2001). Analysis of relative gene expression data using real-time quantitative PCR and the $2-\Delta \Delta \mathrm{CT}$ method. Methods 25:402-408

Ming R, R VanBuren, Y Liu, M Yang, Y Han, LT Li, Q Zhang (2013). Genome of the long-living sacred lotus (Nelumbo nucifera Gaertn.). Genome Biol 4; Article R41

Tan FC, SM Swain (2007). Functional characterization of AP3, SOC1 and WUS homologues from citrus (Citrus sinensis). Physiol Plantarum 131:481-495

Papaefthimiou D, A Kapazoglou, AS Tsaftaris (2012). Cloning and characterization of SOC1 homologs in barley (Hordeum vulgare) and their expression during seed development and in response to vernalization. Physiol Plantarum 146:71-85

Ruokolainen S, YP Ng, VA Albert, P Elomaa, TH Teeri (2011). Overexpression of the Gerbera hybrida At-SOC1-like 1 gene GhSOC1 leads to floral organ identity deterioration. Ann Bot 107:14911499

Wang SL, B Margherita, YM Yang, XX Zhang (2015). Molecular cloning and potential function prediction of homologous SOC1 genes in tree peony. Plant Cell Rep 34:1459-1471

Tamura K, J Dudley, M Nei, S Kumar (2007). MEGA4: Molecular Evolutionary Genetics Analysis (MEGA) software version 4.0. Mol Biol Evol 24:1596-1599

Wei J, D Liu, G Liu, J Tang, Y Chen (2016). Molecular cloning, characterization, and expression of MiSOC1: A homolog of the flowering gene SUPPRESSOR OF OVEREXPRESSION OF CONSTANS1 from Mango (Mangifera indica L). Front Plant Sci 7; Article 1758

Yang M, L Zhu, L Xu, C Pan, Y Liu (2014). Comparative transcriptomic analysis of the regulation of flowering in temperate and tropical lotus (Nelumbo nucifera) by RNA-Seq. Ann Appl Biol 165:73-95

Yu YC, LF Qiao, JC Chen, YH Rong, YH Zhao, XK Cui, JP Xu, XM Hou, CH Dong (2020). Arabidopsis REM16 acts as a B3 domain transcription factor to promote flowering time via directly binding to the promoters of SOC1 and FT. Plant J 103:1386-1398

Zheng YK, MT Liu, CH Jia, JY Wang, BY Xu, ZQ Jin, W Li, JH Liu (2020). Characteristics of banana B genome MADS-box family demonstrate their roles in fruit development, ripening, and stress. Sci Rep 10; Article 20840

Zhong XF, X Dai, J Xv, H Wu, B Liu, H Li (2012). Cloning and expression analysis of GmGAL1, SOC1 homolog gene in soybean. Mol Biol Rep 39:6967-6974 